\title{
Origin, Stemness, Marker and Signaling Pathway of Oral Cancer Stem Cell
}

\author{
Dicha Yuliadewi Rahmawati ${ }^{1}$, Hernindya Dwifulqi ${ }^{2}$, Ferry Sandra ${ }^{3}$ \\ ${ }^{1}$ Department of Oral Biology, Faculty of Dentistry, Maranatha Christian University, Bandung, Indonesia \\ ${ }^{2}$ Department of Dental Materials, Faculty of Dentistry, Maranatha Christian University, Bandung, Indonesia \\ ${ }^{3}$ Department of Biochemistry and Molecular Biology, Faculty of Dentistry, Universitas Trisakti, Jakarta, Indonesia
}

Cancer constitutes of complex heterogeneous organ-like structures with a hierarchical cell structure, and only minor phenotypic subpopulations with stem-like properties have a dual capacity to indefinitely self-renew and generate all heterogeneous cell phenotypes consisting of bulk tumor cells. Cancer stem cells (CSC) has similar properties to ordinary stem cells. It is understood that CSC is responsible for the recurrence of metastasis and drug resistance. Thus, control of CSC can provide successful therapy intervention that inhibits cancer growth and aggressive behavior. Conventional cancer therapy is realized to be insufficient for oral cancer therapy. Meanwhile, accurate targeting of OCSC has proved to be a significant challenge due to the commonality of many markers between OCSC and healthy cells. This article discusses the current understanding of oral CSC, with focus on origin, stemness, marker and signalling pathway.

Keywords: oral cancer stem cell, CSC, marker, origin, stemness, therapy

\section{Introduction}

Cancer is a genetic unstable condition characterized by uncontrolled cell proliferation. Cancer could be caused and powered by aberrant stem cells from normal, tissue-specific stem cells and/or stem cell niches. These tumor-initiating stem cells have properties of self-renewal and the ability to generate progenitor stem cells close to those of their normal stem cells. ${ }^{1}$ Cancer stem cell (CSC) has several main properties, including the ability to indefinitely renew in an undifferentiated state, limitless proliferative potential, toxic xenobiotics resistance, high DNA repair capability, and the

Date of submission: February 4, 2020

Last Revised: May 15, 2020

Accepted for publication: May 15, 2020

\section{Corresponding Author:}

Ferry Sandra

Department of Biochemistry and Molecular Biology, Division of Oral Biology

Faculty of Dentistry, Universitas Trisakti, Jl. Kyai Tapa No.260

Jakarta, Indonesia

E-mail: ferry@trisakti.ac.id ability to drive malignant cell expansion. ${ }^{2}$ Despite sharing features with normal stem cells, the characteristics of selfrenewal and differentiation of CSC lead to cells being deregulated with different stages of differentiation arrest. The level of division in normal stem cell tissues is strongly linked to their functional needs, yet this regulation in CSC is lost. ${ }^{3}$ In a number of solid tumors including breast, prostate, colorectal, pancreatic, brain, and ovarian cancer, CSCs have been identified. ${ }^{3}$

Developing and designing a new method to counter and eradicate CSCs is difficult as they are guarded by structures of resistance that make them less vulnerable to 
traditional therapies. ${ }^{1,3,4}$ Several key signaling pathways have been demonstrated in this regulatory capacity to play important roles. ${ }^{5} \mathrm{CSC}$ is distinguished by a nearly infinite capacity for multiline self-renewal and differentiation while preserving a non-differentiated status. ${ }^{6}$

The presence of oral CSC (OCSC) populations was originally suggested due to an expanding tumor mass can only be formed by a subpopulation of oral squamous cell carcinoma (OSCC) cells. Specific subpopulation of OSCC cells derived from OSCC cell lines was reported to have features of both stem cells and advanced metastatic tumors including self-renewal, tumor capacity, patterns of migration and radioresistance. ${ }^{7}$ Numerous research groups documented that OCSC populations were successfully isolated with various markers. Cell-surface markers or particular functional properties were suggested to distinguish OCSC from OSCC. However, there were some variabilities of OCSC populations. ${ }^{8}$

Studies of many cancer types including OSCC have identified CSCs using specific markers, but it is still unclear. This is compounded further by the presence of multiple subtypes within OSCC, making investigation reliant on the use of multiple markers. OCSC is highly tumorigenic compared to the other oral cancer cells and are believed to be largely responsible for the biological characteristics of cancer, namely, rapid growth, invasion, and metastasis. ${ }^{9}$

\section{Origin of OCSC}

OSCC development demands on a proliferative pattern, the origins of OCSC specific population raise some questions. Does OCSC arise from mutations in the oral mucosa of ordinary somatic stem cells, or are OCSC characteristics developed from genetic alterations? Or more advanced cell dedifferentiation? Given the current confusion about the properties of OCSC, it is not yet possible to exclude. Some comments on the root of OCSC have also been reported. ${ }^{2}$

\section{OCSC from Normal Somatic Stem Cells}

The transformation of a normal human cell into a cancer cell involves 3-6 genetic events. For OSCC, a genetic tumor progression model was proposed, and progressive genetic changes were also found to correlate with phenotypic malignancy progression in OSCC. According to this model, it takes months or years to build most OSCC. Since normal human oral epithelia has an estimated renewal rate of about 14-24 days, most epithelial cells do not exist long enough to accumulate the genetic changes needed to develop an OSCC. The hierarchical stem cell structure present in human oral epithelia indicates that stem cells are the only long-time residents of oral epithelia and, consequently, the only cells able to accumulate the necessary number of genetic changes for malignancy to develop. The derivation of CSCs from their normal tissue counterparts is also supported by several other indirect arguments. For example, CSCs and ordinary somatic stem cells share patterns of molecular expression that are related to mechanisms for regulating stem cell proliferation and differentiation, such as the Notch, Sonic hedgehog (SHH) and Wingless-related integration site (Wnt) signaling pathways. Therefore, it is more likely that a newly emerging OCSC would retain a pre-existing normal stem cell's self-renewal machinery rather than develop new self-renewal pathways. ${ }^{2}$

\section{OCSC from Mature Cells}

Both in vitro and in vivo studies suggest that oncogenicity of keratinocytes can arise and stimulate natural ability to regenerate stem cells and potentially reduce differentiation. If limited progenitors could have the significant potential for stem cell self-renewal, further genetic changes could be developed which would lead to regeneration as well. An in vitro study showed that disrupted DNA could lead to the development of malignant polyploid giant cells that might avoid cell death/differentiation and promoted stem-like cells. This demonstrated a remarkable possible origin for CSC. In culture of senescent breast epithelial cells, a similar process of genomic instability and micronucleation were identified, and binucleated or multi-nucleated cells were resulted in culture. Neosis was identified, and so far only for in vitro environments, and it remains to be determined if it is only an artefact of the conditions of in vitro culture. Giant cells had not been detected in clinical OSCC, although there were documentations of other forms of carcinomas in which they were listed as the host's secondary reactive event rather than neoplastic. ${ }^{2}$

\section{Stemness of OCSC}

A key aspect of CSC is their propensity towards selfrenewal, which appears to play a major role in triggering and preserving the nucleus of cancer cells. Some intracellular signaling pathways including SHH, Notch, Wnt, B-cellspecific Moloney murine leukemia virus integration site 1 (Bmi1), Phosphatase and Tensin homolog (PTEN), Bone 
Morphogenetic Proteins (BMP), and Transforming Growth Factor (TGF)- $\beta$ may maintain self-renewal of CSCs. Among these pathways, there has been detailed evidence of Notch and Bmil functions in oral cancer stemness.

Initiation of the Notch1 signaling pathway is important for the preservation of CSCs. Proinflammatory cytokine $\mathrm{TNF} \alpha$ improves self-renewal capability and tumorigenicity correlated with the stimulation of the Notch pathways. Hes1 is the target of activated Notch1 in TNF $\alpha$-induced OCSC, and its knockdown suppresses the self-renewal potential. Hes1-deficient mice demonstrated early differentiation, loss of progenitor cells and resulting lethality. ${ }^{8}$

\section{OCSC Marker}

OCSC isolation was performed primarily with the Cluster of Differentiation (CD)44 marker that was initially used to isolate CSC from breast cancer. ${ }^{10} \mathrm{CD} 44$ is a large hyaluronic receptor transmembrane glycoprotein on the surface of cells involved in opposing roles in cell migration and adhesion. CD44 has a function as an integral surface molecule capable of dealing with different intrinsic and extrinsic signals to control a range of gene expressions. CD44 was highly expressed in oral epithelial carcinoma cells, in situ carcinoma, and some infiltrating lymphocytes. ${ }^{8}$

CD133 was also reported as an OCSC marker. The $\mathrm{CD}_{133^{+}}$cells showed decreased paclitaxel resistance. ${ }^{11}$ $\mathrm{CD}_{133^{+}}$cell expressed higher levels of stem genes, positive spheroid development, heterogeneous tumor formation, and increased clonogenicity from OSCC cell lines (1-2\%) as well as from human OSCC samples (1-3\%). Recent studies have found a correlation between CD133 expression and cancer stage. Expression of CD133 was higher in cancer stage III and IV than stage I and II.

Besides CD44 and CD133, CD10 was reported to be correlated with OCSC since $\mathrm{CD} 10^{+}$head and neck cancer (HNC) cells showed high expression of Oct $3 / 4$, a pluripotent stem cell marker. The $\mathrm{CD} 10^{+}$cells were fairly immune to cisplatin and radiation, and had tumorigenic sphere-forming and malignant properties, indicating that $\mathrm{CD} 10$ could be a marker of OCSC as well. ${ }^{9}$

Aldehyde Dehydrogenase (ALDH) has been used as a marker in OCSC. ALDH is a cytosolic isoenzyme that oxidizes intracellular aldehydes and thus corresponds to the oxidation of retinol to retinoic acid in initial differentiation of stem cells. ${ }^{9} \mathrm{ALDH}^{+}$cells demonstrated plasticity with the ability to form tumor spheres in serum-free media and the ability to generate $\mathrm{ALDH}^{-}$cells in vitro. Although there are many ALDH isoforms, ALDH1 is particularly important in OCSC. In addition, $\mathrm{ALDH}^{+}$oral leukoplakia was more likely to develop OSCC. ${ }^{11}$

OSCC-derived tumor spheres showed enhanced stemlike properties and expressed higher levels of pluripotent transcription factors such as Oct4, Klf4, Lin28, Nanog and Sox2. Sphere-forming oral cancer cells also exhibit substantial expression of OCSC-specific markers such as CD44 and ALDH1. ${ }^{12}$ Table 1 shows the summary of the OCSC markers' role.

\section{Targeting OCSC}

Within the large and heterogeneous population of cells comprising the mass of $\mathrm{HNC}$, the small subpopulation of $\mathrm{CSC}$ may be responsible for cancer recurrence and metastasis initiation due to high migration capacity and resistance to both radio and chemotherapy. The main challenge in CSCtargeting therapy is the heterogeneity of CSCs. ${ }^{17}$ Some CSCs were identified via different surface markers, however, and there are no general markers available for the global recognition of these cells. ${ }^{18}$

\section{Targeting CSC Cell Surface Markers}

CSCs in various cancers express specific surface markers, such as CD133 in hepatocellular and gastric CSCs, CD50, CD9, CD24, and CD26 in human malignant mesothelioma CSCs, CD44, CD24, and Epithelial Surface Antigen (ESA),

Table 1. Role of the OCSC markers. ${ }^{13-16}$

\begin{tabular}{ll}
\hline \multicolumn{1}{c}{ Roles } & \multicolumn{1}{c}{ Markers } \\
\hline Reprogramming into pluripotent cell & Oct4 and Nanog \\
Regulating self-renewal & Oct4, Nanog, Sox2, c-Met and ALDH \\
Maintaining undifferentiated state & Oct4, Nanog, Sox2, CD44 and Bmi1 \\
Increasing tumor sphere formation and tumorigenicity & CD44, SLC2A13 and CD133 \\
\hline
\end{tabular}


in pancreatic CSCs. Among these markers, CD133 is considered as one of the most important CSC associated marker identified so far. Expression of CSC markers in oral cavity was still poorly investigated in the population of Indonesia. So far, there was a report showing that expression of CD133 was increased in the peripheral blood sample of oral premalignant lesions. ${ }^{19}$ In addition, there are preclinical studies with an emphasis on CD44 to verify the efficacy and toxicity of CSC targeting agents. A Phase I single-dose escalation trial for patients with advanced $\mathrm{HNC}$ with antiCD44 bivatuzumab mertansine was reported..$^{20}$

\section{Targeting CSC Environment}

CSC environment consists of different components including cancer-associated fibroblast, immune cells, multipotent stromal cells, endothelial and perivascular cells and their mysterious factors, including growth factors and cytokines. Tumor stroma is able to produce and retain CSC, shield the tumor from the immune system, and contribute to Epithelial Mesenchymal Transformation (EMT) activation, contributing to increased tumor progression, invasion, and secondary tumor recolonization. ${ }^{18}$

Vascular endothelial cell, a type of CSC niche stromal cell required for angiogenesis, can also secrete growth factors and cytokines that enhance CSC proliferation and promote the maintenance of CSC properties in HNC. In theory, interfering with the growth and survival of vascular endothelial cell could inhibit not only angiogenesis but also CSC self-replication. VEGF is a dominant cancer cellsecreted proangiogenic factor, a well-recognized therapeutic target. Different angiogenic inhibitors have been developed that can also inhibit the self-regeneration of CSCs leading to lower tumor growth. Bevacizumab has been shown to inhibit CSC populations in Non-small-cell lung carcinoma (NSCLC) in combination with anti-hepatoma-derived growth factor (HDGF) antibody. Nevertheless, inhibition of VEGFR in breast cancer will increase the population of CSCs by inducing hypoxia. Using a VEGFR inhibitor in conjunction with HIF inhibition in combination therapy may provide a more effective treatment strategy to address this. 18

\section{Immunotherapy}

Immunotherapy is an emerging field that can abrogate CSC's ability to reinitiate. ${ }^{18}$ Numerous approaches to immunotherapy have been developed to combat $\mathrm{HNC}$, including vaccines, T-cell infusion, immune control point inhibitors and monoclonal antibodies. Food and Drug Administration (FDA) of The United States of America (USA) approved Nivolumab and Pembrolizumab, antiProgrammed Death-1 (PD-1) antibodies, in the secondline setting for HNC. Results of the study showed that Nivolumab and Pembrolizumab could enhance overall survival compared to conventional chemotherapy. Nevertheless, Nivolumab and Pembrolizumab response rates in $\mathrm{HNC}$ remained low, ranging from only 13 to $20 \%$, though survival improved in 1 of 10 patients receiving these therapies. ${ }^{21}$

\section{Targeting Second Messenger}

Recent studies have suggested that $\mathrm{Ca}^{2}$ signaling is important in regulating the stemness of oral cancer. $\mathrm{Ca}^{2+}$ is an universal second messenger which regulates many physiological processes. Homeostasis of $\mathrm{Ca}^{2+}$ is disrupted in carcinogenesis, leading to fail in regulation of cell proliferation, migration and suppression of apoptosis. ${ }^{20}$ Therefore cancer stem cell properties can be regulated by $\mathrm{Ca}^{2+}$ channels and signals. ${ }^{22}$

$\mathrm{Ca}^{2+}$ influx is tightly controlled by the Store-operated $\mathrm{Ca}^{2+}$ Entry (SOCE) pathway and mediated by the $\mathrm{Ca}^{2+}$ Release-activated Channels (CRAC). Cells release $\mathrm{Ca}^{2+}$ from the endoplasmic reticulum (ER) after stimulation, accompanied by the influx of extracellular $\mathrm{Ca}^{2+}$ by SOCE. Not only does SOCE refill the depleted ER $\mathrm{Ca}^{2+}$ stocks, It also provides a direct $\mathrm{Ca}^{2+}$ signal allowing downstream responses including a signaling route for the stimulated T-cell nuclear factor (NFAT). ${ }^{22}$

\section{Conclusion}

OCSC has features of both stem cells and advanced metastatic cancer cells including self-renewal, migration, tumor formation, chemoresistance and radioresistance. OCSC can be derived from normal somatic stem cells and mature cells. OCSC markers could be used as for targeting OCSC. CD133 could be a promising target in OCSC.

\section{References}

1. Wijaya L, Agustina D, Lizandi AO, Kartawinata MM, Sandra F. Reversing breast cancer stem cell into breast somatic stem cell. Curr Pharm Biotechnol. 2011; 12(2): 189-95.

2. Costea DE, Tsinkalovsky O, Vintermyr OK, Johannessen AC, Mackenzie IC. Cancer stem cells - New and potentially important targets for the therapy of oral squamous cell carcinoma. Oral Dis. 2006; 12(5): 443-54. 
3. Aguilar-Gallardo C, Rutledge EC, Martínez-Arroyo AM, Hidalgo JJ, Domingo S, Simón C. Overcoming challenges of ovarian cancer stem cells: novel therapeutic approaches. Stem Cell Rev Rep. 2012; 8(3): 994-1010.

4. Sandra F. A Brief outlook on pharmacogenetics (PGx): Focus in microRNAs (miRNAs) and cancer stem cells (CSCs). Indones J Cancer Chemoprevent. 2019; 10(1): 46-50.

5. Matsui WH. Cancer stem cell signaling pathways. Medicine. 2016; 95(1 Suppl 1): S8-19.

6. Rao CV, Mohammed A. New insights into pancreatic cancer stem cells. World J Stem Cells. 2015; 7(3): 547-55.

7. Chiou SH, Yu CC, Huang CY, Lin SC, Liu CJ, Tsai TH, et al. Positive correlations of Oct-4 and Nanog in oral cancer stem-like cells and high-grade oral squamous cell carcinoma. Clin Cancer Res. 2008; 14(13): 4085-95.

8. Shin KH, Kim RH. An updated review of oral cancer stem cells and their stemness regulation. Crit Rev Oncog. 2018; 23(3-4): 189-200.

9. Baillie R, Tan ST, Itinteang T. Cancer stem cells in oral cavity squamous cell carcinoma: A review. Front Oncol. 2017; 2(7): 112. doi: 10.3389/fonc.2017.00112.

10. Rodini CO, Lopes NM, Lara VS, Mackenzie IC. Oral cancer stem cells - Properties and consequences. J Appl Oral Sci. 2017; 25(6): 70815.

11. Chen YT, Chong YM, Cheng CW, Ho CL, Tsai HW, Kasten FH, et al. Identification of novel tumor markers for oral squamous cell carcinoma using glycoproteomic analysis. Clin Chim Acta. 2013; 420: 45-53.

12. Ren ZH, Zhang CP, Ji T. Expression of SOX2 in oral squamous cell carcinoma and the association with lymph node metastasis (Review). Oncol Lett. 2016; 11(3): 1973-9.

13. Chiou SH, Yu CC, Huang CY, Lin SC, Liu CJ, Tsai TH, et al. Positive correlations of Oct- 4 and Nanog in oral cancer stem-like cells and high-grade oral squamous cell carcinoma. Clin Cancer Res. 2008; 14(13): 4085-95.

14. Shibata M, Hoque MO. Targeting cancer stem cells: A strategy for effective eradication of cancer. Cancers. 2019; 11(5): 732. doi: $10.3390 /$ cancers 11050732 .

15. Patel SS, Shah KA, Shah MJ, Kothari KC, Rawal RM. Cancer stem cells and stemness markers in oral squamous cell carcinomas. Asian Pac J Cancer Prev. 2014; 15(20): 8549-56.

16. Peitzsch C, Nathansen J, Schniewind SI, Schwarz F, Dubrovska A. Cancer stem cells in head and neck squamous cell carcinoma: Identification, characterization and clinical implications. Cancers. 2019; 11(5): E616. doi: 10.3390/cancers11050616.

17. Kuhlmann JD, Hein L, Kurth I, Wimberger P, Dubrovska A. Targeting cancer stem cells: promises and challenges. Anticancer Agents Med Chem. 2016;16(1): 38-58.

18. Sun HR, Wang S, Yan SC, Zhang Y, Nelson PJ, Jia HL, et al Therapeutic strategies targeting cancer stem cells and their microenvironment. Front Oncol. 2019; 9: 1104. doi: 10.3389/ fonc.2019.01104.

19. Amtha R, Gunardi I, Sandra F, Ernawati DS. Expression of CD133 in various premalignant and proliferative lesions. Dent J (Maj Ked Gigi). 2015; 48(2): 64-8.

20. Tijink BM, Buter J, de Bree R, Giaccone G, Lang MS, Staab A, et $a l$. A phase I dose escalation study with anti-CD44v6 bivatuzumab mertansine in patients with incurable squamous cell carcinoma of the head and neck or esophagus. Clin Cancer Res. 2006; 12(20 Pt 1): 6064-72.

21. Gavrielatou N, Doumas S, Economopoulou P, Foukas PG, Psyrri A. Biomarkers for immunotherapy response in head and neck cancer. Cancer Treat Rev. 202; 84:101977. doi: 10.1016/j.ctrv.2020.101977.

22. Terrié E, Coronas V, Constantin B. Role of the calcium toolkit in cancer stem cells. Cell Calcium. 2019; 80: 141-51. 